\section{Are occupational, hobby, or lifestyle exposures associated with Philadelphia chromosome positive chronic myeloid leukaemia?}

J Björk, M Albin, H Welinder, H Tinnerberg, N Mauritzson, T Kauppinen, U Strömberg, B Johansson, R Billström, Z Mikoczy, T Ahlgren, P-G Nilsson, F Mitelman, L Hagmar

\begin{abstract}
Objectives-To investigate a broad range of occupational, hobby, and lifestyle exposures, suggested as risk factors for Philadelphia chromosome positive $(\mathbf{P h}+)$ chronic myeloid leukaemia (CML).

Methods-A case-control study, comprising $255 \mathrm{Ph}+\mathrm{CML}$ patients from southern Sweden and matched controls, was conducted. Individual data on work tasks, hobbies, and lifestyle exposures were obtained by telephone interviews. Occupational hygienists assessed occupational and hobby exposures for each subject individually. Also, occupational titles were obtained from national registries, and group level exposure-that is, the exposure proportion for each occupational title-was assessed with a job exposure matrix. The effects of 11 exposures using individual data and two exposures using group data (organic solvents and animal dust) were estimated.
\end{abstract}

Results-For the individual data on organic solvents, an effect was found for moderate or high intensity of exposure (odds ratio (OR) $3.4,95 \%$ confidence interval $(95 \% \mathrm{CI}) 1.1$ to 11$)$ and for long duration (15-20 years) of exposure (OR $2.1,95 \%$ CI 1.1 to 4.0$)$. By contrast, the group data showed no association (OR $0.69,95 \%$ CI 0.27 to 1.8 ; moderate or high intensity versus no exposure). For extremely low frequency electromagnetic fields (EMFs), only individual data were available. An association with long occupational exposure to EMFs was found (OR $2.3,95 \%$ CI 1.2 to 4.5 ). However, no effect of EMF intensity was indicated. No significant effects of benzene, gasoline or diesel, or tobacco smoking were found. OR estimates below unity were suggested for personal use of hair dye and for agricultural exposures.

Conclusions-Associations between exposure to organic solvents and EMFs, and $\mathrm{Ph}+\mathrm{CML}$ were indicated but were not entirely consistent.

(Occup Environ Med 2001;58:722-727)

Keywords: risk factors; epidemiology; case-control study

Correspondence to:

Dr J Björk

jonas.bjork@ymed.lu.se

Accepted 4 July 2001

Chronic myeloid leukaemia (CML) is morphologically and cytogenetically homogenous, with
Main messages

- Some limited evidence of an association between long time exposure to organic solvents and extremely low frequency electromagnetic fields, respectively, and the risk of Philadelphia chromosome positive chronic myeloid leukaemia in adults were found.

- No harmful effects of gasoline or diesel, tobacco smoking, personal use of hair dye, and agricultural exposures with respect to Philadelphia chromosome-positive chronic myeloid leukaemia in adults were found.

almost all of the cases being Philadelphia chromosome positive $(\mathrm{Ph}+)$ - that is, having the translocation $\mathrm{t}(9 ; 22)(\mathrm{q} 34 ; \mathrm{q} 11)$ that results in the $B C R / A B L$ fusion gene. ${ }^{1}$ The aetiology is poorly known. Studies of Japanese atom bomb survivors exposed to ionising radiation have shown an excess risk of CML. ${ }^{2}$ Other exposures that have been suggested as risk factors include treatment with DNA topoisomerase II inhibitors, tobacco smoking, personal use of hair dye, benzene and other organic solvents, extremely low frequency electromagnetic fields (EMFs), viruses, and pesticides. ${ }^{3-11}$

The aim of the present case-control study was to investigate a broad range of occupational, hobby, and lifestyle exposures, suggested as risk factors for CML, from both individual exposure data assessed by occupational hygienists and group level exposure data assessed by a job exposure matrix.

\section{Materials and methods}

CASES

The study is based on a series of 255 adult patients with $\mathrm{Ph}+\mathrm{CML}$ from southern Sweden, cytogenetically analyzed 1976-93 at the Department of Clinical Genetics, Lund, Sweden. ${ }^{12}$ The Department performs cytogenetic investigations on suspect CML from the catchment areas of Lund University Hospital, as well as on patients from other parts of southern Sweden. All referrals for cytogenetic investigations are made within the public health care system. In the catchment areas of Lund University Hospital, referrals for cytogenetic investigations were routinely used in the diagnostic evaluation of haematological malignancies throughout the study period, implying 
Table 1 Descriptive statistics for Ph+CML cases and controls

\begin{tabular}{lll}
\hline & Cases & Controls \\
\hline Eligible (n) & 255 & $765^{\star}$ \\
Contacted for interview (n) & 255 & $349 \dagger$ \\
Interviewed (n) & 226 & 251 \\
Next of kin interview (n (\%)) & $182(81)$ & $35(14)$ \\
Men (n (\%)) & $125(55)$ & $140(56)$ \\
Year of birth (median $(10 \%-90 \%)) \ddagger$ & $1935(1913-54)$ & $1934(1913-55)$ \\
Age (median $(10 \%-90 \%)) \ddagger 5$ & $51(31-71)$ & $52(31-72)$ \\
Year of diagnosis (median $(10 \%-90 \%)) \ddagger$ & $1986(1978-92)$ & \\
\hline
\end{tabular}

*Three controls were selected for each case, matched for sex, age, and county of living, and were obtained from the study population of southern Sweden for the calendar year in which each case was diagnosed.

tWhen interview information for a control could not be obtained, another control from the matched set was contacted.

$\ddagger$ Median (10th-90th percentiles).

SAge at the year of diagnosis for cases. Age at the year of selection from the study population for controls.

for other parts of southern Sweden is less complete, but has improved during the study period. The number of referrals of Philadelphia chromosome-negative CML to Lund was too few (seven patients during 1976-93) to be studied.

\section{CONTROLS}

The Swedish national bureau of statistics (Statistics Sweden) selected three controls for each case, matched with respect to sex, age, and county of living from the study population of southern Sweden at the calendar year each case was diagnosed. The study aimed at collecting registry data for all controls, whereas interviews were restricted to one randomly selected control in each matched set. However, when interview information for a control could not be obtained, another control from the matched set was selected.

STRUCTURED TELEPHONE INTERVIEWS

All eligible cases together with the selected controls were retrospectively contacted 1995-7 with a letter describing the purpose and the main questions to be asked during the structured telephone interview. If the subject was dead or too ill to participate, a next of kin was selected in the following order: spouse, parent, sibling, and child. The participation rate among contacted cases and controls were $89 \%$ and $72 \%$, respectively, resulting in 226 interviewed cases and 251 controls. Information had to be obtained from next of kin much more often for cases than controls (table 1). Among next of kin, spouses, and children were most often interviewed $(49 \%$ and $33 \%$, respectively).

The structured telephone interviews were conducted by one of three occupational health nurses, who for ethical reasons were aware of the case-control status. A lifelong occupational history was obtained, focusing on all jobs held for at least 1 year, including work task, department, and name of the company. No specific exposures were asked for in the interview, but follow up questions were triggered about application of pesticides by working as a gardener or horticulturist, lumberjack, railway worker (track maintenance), farmer, or farmhand; type of cargo by working as a driver or on a merchant ship; handling of cytostatics by working as a nurse; and working at a meat counter by work as a shopkeeper or shop assistant. Information on extra jobs held for at least 1 year was collected in the same way as for the main job. The following hobby activities were explicitly asked for: motor repair, painting with oil, furniture renovation, construction of models, riding, animal breeding, hunting, and gardening. For each hobby, information on the duration and the average hours a week spent on the activity was collected. Furthermore, questions were asked about regular personal use of hair dye and smoking habits (smoking type, duration, and average consumption). The questions on medical history before the time of the case diagnosis focused on chemotherapy and radiotherapy as well as on treatment with isotopes.

INDIVIDUAL EXPOSURE ASSESSMENTS

For a broad range of occupational and hobby exposures (table 2), individual exposure assessments were performed by one of three occupational hygienists, on the basis of the structured telephone interviews made by the occupational health nurses. The occupational hygienists were unaware of the case-control status. The assessments for both cases and controls were restricted to exposure periods of at least 1 year during the 20 years before the diagnosis of the case. One of the hygienists (HW) supervised the assessments.

Quantitative definitions of the different exposure intensity categories (low, moderate, and high) were used for organic solvents, benzene, and EMFs. For organic solvents, these categories corresponded to $1-5 \%,>5-15 \%$, and $>15 \%$, respectively, of the recent Swedish occupational exposure limit values. For benzene, the corresponding categories were $0.05-$ $0.2 \mathrm{mg} / \mathrm{m}^{3},>0.2-0.5 \mathrm{mg} / \mathrm{m}^{3}$, and $>0.5 \mathrm{mg} / \mathrm{m}^{3}$. Exposure to EMFs was classified according to 8 hour arithmetic means reported for different occupations, ${ }^{13}$ with the following categories: $0.23-0.30 \mu \mathrm{T},>0.30-0.50 \mu \mathrm{T}$, and $>0.50 \mu \mathrm{T}$. For all other occupational exposures, the exposure intensity categories were demarcated by examples of occupations and tasks. Exposures to cattle or poultry - that is, animal dust - and fresh meat were considered as proxy variables for exposure to animal borne viruses. The main occupation associated with such exposures was farming. The confidence in classification of the occupational exposure was assessed (low or high confidence) based on the quality of the information on work tasks and exposures from the interviews, as well as on the likelihood of exposure for different work tasks. Hobby exposure was assessed for all occupational agents, except EMFs, ionising radiation, and cytostatics. For regular personal use of hair dye and smoking habits, the classification was directly based on the specific questions in the interview. Subjects were classified as smokers if they had smoked at least one cigarette a day (or an equivalent amount for other smoking types ${ }^{14}$ ) for at least 1 year during the 20 years before the diagnosis of the case. 
Table 2 Effects of occupational, hobby, and lifestyle exposures, assessed for each subject individually, 20 years or less before diagnosis on the risk for Ph+ $C M L$

\begin{tabular}{|c|c|c|c|c|c|c|c|c|}
\hline \multirow[b]{2}{*}{ Exposure/intensity } & \multicolumn{3}{|l|}{ Cases (n) } & \multicolumn{3}{|c|}{ Controls (n) } & \multirow[b]{2}{*}{$O R$} & \multirow[b]{2}{*}{$95 \% C I$} \\
\hline & Exposed & Unexposed & Uncertain & Exposed & Unexposed & Uncertain & & \\
\hline Organic solvents & 55 & 146 & 25 & 51 & 175 & 25 & 1.4 & 0.85 to 2.3 \\
\hline Hobby/low & 43 & & & 44 & & & 1.2 & 0.70 to 2.1 \\
\hline Moderate/high & 12 & & & 7 & & & 3.4 & 1.1 to 11 \\
\hline Gasoline or diesel fuel & 35 & 185 & 6 & 41 & 206 & 4 & 1.0 & 0.59 to 1.7 \\
\hline Gasoline or diesel exhaust gases & 39 & 181 & 6 & 43 & 193 & 15 & 1.1 & 0.64 to 1.9 \\
\hline Benzene & 24 & 190 & 12 & 23 & 215 & 13 & 1.2 & 0.66 to 2.3 \\
\hline Pesticides & 22 & 197 & 7 & 31 & 204 & 16 & 0.75 & 0.42 to 1.3 \\
\hline Cattle or poultry & 21 & 204 & 1 & 38 & 212 & 1 & 0.63 & 0.35 to 1.1 \\
\hline Fresh meat & 23 & 198 & 5 & 44 & 204 & 3 & 0.55 & 0.31 to 1.0 \\
\hline Fresh wood & 11 & 210 & 5 & 20 & 227 & 4 & 0.68 & 0.32 to 1.5 \\
\hline Extremely low frequency electromagnetic fields ${ }^{\star}$ & 55 & 151 & 20 & 49 & 182 & 20 & 1.7 & 1.0 to 2.8 \\
\hline Low & 25 & & & 22 & & & 2.0 & 1.0 to 4.1 \\
\hline Moderate & 22 & & & 16 & & & 1.6 & 0.77 to 3.4 \\
\hline High & 8 & & & 11 & & & 1.2 & 0.44 to 3.1 \\
\hline Tobacco smoking & 81 & 131 & 14 & 101 & 144 & 6 & 0.81 & 0.53 to 1.2 \\
\hline Regular personal use of hair dye & 25 & 195 & 6 & 51 & 193 & 7 & 0.35 & 0.18 to 0.68 \\
\hline
\end{tabular}

${ }^{\star}$ Only occupational exposure was assessed.

\section{REGISTRY DATA}

Occupational titles were obtained for $99 \%$ of the eligible cases and controls from the National Swedish censuses for every 5 th year during the period 1960-90 except 1965 and were recoded to the 1980 classification scheme as suggested by Statistics Sweden.

GROUP LEVEL EXPOSURE ASSESSMENT

We used a Finnish job exposure matrix $\left(\right.$ FINJEM) ${ }^{15}$ to assess the proportion of workers exposed above a minimum level of intensity for each occupational title (occupational group). Such a proportion may also be interpreted as the estimated exposure probability for a randomly selected group member. FINJEM presents exposure probabilities and average intensity levels for the calendar periods 1945-59, 1960-84, and 1985-94 for Finnish occupational groups, which were translated to the Swedish 1980 classification scheme by the occupational hygienists. For each subject, the probability of no exposure was calculated as the product of one minus the exposure probability of the subject's occupational group for every 5 th year available during the 20 year time window before the year of diagnosis. The exposure probability was then defined as one minus the probability of no exposure.

Two occupational exposures, organic solvents and animal dust, for which the corresponding odds ratio (OR) estimates in the analysis of individual exposure data differed markedly from unity (table 2), were assessed on the group level. Exposure data for EMFs in FINJEM are incomplete before 1985 and could therefore not be used. In FINJEM, the intensity level for organic solvents is a weighted average of four classes of solvents. To facilitate comparisons with moderate or high intensity of occupational exposure to organic solvents in the individual assessment, $5 \%$ of the Swedish occupational exposure limit value for toluene (50 ppm) was used as minimum intensity. Thus, only exposure probabilities for occupational groups in FINJEM with an average intensity above $2.5 \mathrm{ppm}$ were used in the calculations.

The definition of exposure to animal dust in FINJEM is broader than cattle or poultry of the individual exposure assessment. The minimum intensities $\left(0.1 \mathrm{mg} / \mathrm{m}^{3}\right.$ in FINJEM) are, however, roughly comparable. Except for animal dust, exposures with OR estimates below or close to unity in the individual level analysis were not considered for group level analysis.

STATISTICAL ANALYSIS

Individual and group exposure data were analyzed separately. In the individual analysis, conditional logistic regression was used to obtain effect estimates. ${ }^{16}$ Matched sets with identical values for all matching factors were combined for efficiency reasons. ${ }^{17}$ To reduce exposure misclassification for occupational exposures, only exposure assessments with high confidence were used in the analyses. Subjects were classified as unexposed for a particular occupational agent only if they were unexposed with high confidence during the entire assessment period. Subjects were classified as exposed if they were exposed with high confidence for at least 1 year during the assessment period; those who could not be classified as exposed or unexposed with high confidence were excluded from the analyses. Hobby exposure was regarded as equivalent to low intensity occupational exposure.

In the group analysis, conditional binary regression was used to obtain effect estimates under the linear odds ratio (OR) $\operatorname{model}^{18}$ :

$$
O R(x)=1+\beta x
$$

where $\beta$ is the regression parameter and $x$ the exposure probability. The OR estimate was obtained as:

$\hat{O} R=\hat{O} R(x=1)=1+\hat{\beta}$,

where $\hat{\beta}$ is the maximum likelihood estimate of $\beta$. Notice that this effect estimator, derived from group data, is comparable with the OR estimator used for individual data. For the log transformed OR, the $95 \%$ confidence interval (95\% CI) was calculated as ${ }^{19}$ :

$\exp (\ln (1+\hat{\beta}) \pm 1.96 \sqrt{\operatorname{var}(\ln (1+\hat{\beta})})$ 
Table 3 Categorical analysis of the effect of duration of exposure, irrespective of intensity, to organic solvents and extremely low frequency magnetic fields, assessed for each subject individually, on the risk for $\mathrm{Ph}+\mathrm{CML}$

\begin{tabular}{|c|c|c|c|c|}
\hline Exposure and duration & Cases (n) & Controls (n) & $O R$ & $95 \% C I$ \\
\hline \multicolumn{5}{|l|}{ Organic solvents $(\mathrm{y}):^{\star}$} \\
\hline Zero & 146 & 175 & 1.0 & \\
\hline $1-7$ & 9 & 13 & 0.88 & 0.34 to 2.3 \\
\hline $8-14$ & 11 & 13 & 1.0 & 0.41 to 2.3 \\
\hline $15-20$ & 35 & 25 & 2.1 & 1.1 to 4.0 \\
\hline Uncertain & 25 & 25 & 1.2 & 0.65 to 2.1 \\
\hline \multicolumn{5}{|c|}{ Electromagnetic fields $(y): \dagger$} \\
\hline Zero & 151 & 182 & 1.0 & \\
\hline $1-7$ & 10 & 13 & 1.1 & 0.44 to 2.6 \\
\hline $8-14$ & 10 & 14 & 0.83 & 0.33 to 2.1 \\
\hline $15-20$ & 35 & 22 & 2.3 & 1.2 to 4.5 \\
\hline Uncertain & 20 & 20 & 1.4 & 0.71 to 2.7 \\
\hline
\end{tabular}

* Subjects with both hobby and occupational exposure were classified according to the exposure of longest duration.

†Only occupational exposure was assessed.

where

$\operatorname{var}(\ln (1+\hat{\boldsymbol{\beta}})) \approx \frac{\operatorname{var}(\hat{\boldsymbol{\beta}})}{(1+\hat{\boldsymbol{\beta}})^{2}}$.

In the group analysis of organic solvents, 14 cases and 25 controls with non-zero exposure probabilities in FINJEM at an mean intensity consistently below our minimum for the moderate intensity category $(2.5 \mathrm{ppm})$ were excluded.

\section{Results}

INDIVIDUAL ANALYSIS

The relative risk (OR) estimates for $\mathrm{Ph}+\mathrm{CML}$ were significantly above unity for moderate or high intensity of exposure to organic solvents and for any exposure to EMFs (table 2). For EMFs, no consistent relation between the intensity of exposure and the risk for $\mathrm{Ph}+\mathrm{CML}$ was indicated. Excluding subjects with a history of chemotherapy or radiotherapy altered these estimates only marginally (data not shown). Moreover, restricting the analyses to in person interviews (40 matched sets) reduced the precision substantially-for example, the OR estimate for moderate or high intensity of exposure to organic solvents was 8.6 (95\% CI 1.0 to 76$)$. The OR estimate for moderate or high intensity of exposure to organic solvents remained significantly increased when adjusting for exposure to EMFs and vice versa (data not shown). Small numbers made it impossible to investigate reliably effect modification between the two exposures; it is, however, worth noting that five subjects were exposed to both $\mathrm{EMF}$ of any intensity and to organic solvents at moderate or high intensity, all of them cases. The OR estimates for benzene and gasoline or diesel (fuel and exhaust gases) were close to unity for all categories of intensity (overall estimates presented in table 2). The risk estimates were below unity for pesticides, fresh meat, cattle or poultry, fresh wood, regular personal use of hair dye, and tobacco smoking. Furthermore, no relation between cumulative smoking dose (pack-years), and risk of disease was found (data not shown). The numbers of subjects occupationally exposed to ionising radiation (two cases, no controls) or cytostatics (one control, no cases) were too low to permit analyses. In the analyses of duration of exposure to organic solvents and EMFs, effects of exposure were found in the long duration category (15-20 years of exposure, table 3) for both agents.

\section{GROUP ANALYSIS}

In the group analysis of the same subjects as in the individual analysis - that is, participants of the interviews - the OR for Ph+CML for moderate or high intensity of exposure versus no occupational exposure was 0.97 (95\% CI 0.30 to 3.1) for organic solvents. Adding group exposure data for non-participants had no bearing on the risk estimate, whereas adding the two extra controls for each case improved precision (OR $0.69,95 \%$ CI 0.27 to 1.8 ). For animal dust, the OR for Ph+CML for any versus no occupational exposure was below unity, irrespective of control group used (OR 0.39 (95\% CI 0.18 to 0.87 ), using all available group data).

\section{Discussion}

To the best of our knowledge, the present study is the largest and most detailed epidemiological investigation of Ph+CML to date. The salient finding of the individual analysis was the indication that exposure to organic solvents at moderate or high intensity or at long durations may be a risk factor for Ph+CML. Similar associations have recently been reported in a French study of $\mathrm{CML}^{11}$ and in a study of acute myeloid leukaemia (AML) in southern Sweden, using an identical structured telephone interview. ${ }^{20}$ By contrast, a previous report on CML, which did not stratify by intensity or duration of exposure, failed to show such an association. ${ }^{7}$ Assessing the combined effect of intensity and duration of exposure to organic solvents was impossible due to the few subjects in the moderate or high intensity category. However, there was no correlation between intensity and duration among subjects occupationally exposed to organic solvents (Spearman's rank correlation 0.04).

No effect of exposure to benzene or gasoline or diesel on the risk for $\mathrm{Ph}+\mathrm{CML}$ was found. This agrees well with a combined cohort study of petroleum workers who were exposed to petrochemicals-such as benzene at low concentrations and gasoline. ${ }^{6}$ At higher exposures, non-significant effects of occupational exposure to benzene on leukaemias other than AML have, however, been reported. ${ }^{5}$

Exposure to EMFs is prevalent and has therefore potentially a high aetiological explanatory value, but in the present study no clear cut pattern between exposure to EMFs and risk of $\mathrm{Ph}+\mathrm{CML}$ emerged. A recent metaanalysis also failed to show a clear association between EMFs and CML. ${ }^{8}$ In the present study, the overall risk estimate and the risk estimate for long durations of exposure were both increased, whereas no trend with exposure intensity was indicated. The classification of EMF intensity was largely based on the average intensities for various occupational groups reported by Floderus et al. ${ }^{13}$ The number of personal measurements in each occupational group was limited and showed 
large variations in intensity, which implies that misclassification of the intensity of EMFs cannot be ruled out. Non-differential misclassification of a polychotomous exposure variable may attenuate, change the shape, or even reverse the direction of a trend. ${ }^{21}$ Unfortunately, incomplete EMFs data in FINJEM made comparisons with a group analysis impossible. Only occupational exposure to EMF, was assessed. The presented ORs associated with EMFs are relative a background level below $0.23 \mu \mathrm{T}$. Few people in Sweden have residential exposure above this level. In a recent pooled analysis of residential exposure to EMFs and childhood leukaemia, $92 \%$ of the Swedish children were living in homes with exposures below $0.20 \mu \mathrm{T} .^{22}$ Thus, misclassification of EMF intensity due to unmeasured residential exposure was unlikely.

Contrary to previous case-control studies of myelodysplastic syndromes and AML in southern Sweden, ${ }^{23}{ }^{24}$ we saw no effect of cumulative smoking dose on risk of CML. Similar findings have been reported by others. ${ }^{411}$ Thus, the suggestion that tobacco smoking is a risk factor for myeloid leukaemia in general ${ }^{25-28}$ may not be applicable for $\mathrm{Ph}+\mathrm{CML}$, stressing the need for including detailed morphological and genetic features in epidemiological investigations of leukaemias. An OR estimate significantly below unity was found for regular personal use of hair dye. We have no explanation for this except that it is a random finding. No apparent association between use of hair dye and risk of CML was found in another report. ${ }^{4}$

The association between farming and leukaemia in general, which has been studied in numerous epidemiological settings, is likely to be weak, if present at all. ${ }^{29}$ If anything, our data indicated decreased risks of CML associated with agricultural life, manifested by OR estimates below unity for typical agricultural exposures-such as cattle or poultry, fresh wood, and pesticides in the individual analysis.

The results of the individual and group analyses were consistent for cattle or poultry (animal dust) but not for organic solvents. The precision of the group estimates was generally poor compared with the corresponding individual estimates but can be improved by adding extra controls, usually at low cost. ${ }^{1930}$ The group estimate for moderate or high intensity of exposure to organic solvents confidently ruled out a relative risk above 2 when all available controls were used. No bias results from low specificity of a probabilistic JEM if the linear OR model is used. ${ }^{18}$ Bias instead occurs when the estimated exposure probabilities differ from the true exposure prevalencesfor example, if exposed subjects exist in occupational groups with zero probability of exposure. ${ }^{19}$ In the present study, among subjects classified as exposed to organic solvents with moderate or high intensity in the individual exposure assessments (table 2), five of the 12 cases (but none of the controls) had zero probability of exposure according to the JEM. Such discrepancies of the exposure assessments were the main explanation for the difference between the OR estimates of the individual and the group analyses. Other possible sources of errors in the group assessments include the general limitation of registry data to every 5 th year and the translation of FINJEM to Swedish conditions. It may be argued that the group analyses, based on registry data and exposure probabilities, were too crude for less prevalent exposures. On the other hand, such a partially ecological case-control approach ${ }^{19}$ is free from differential biases and thus provides a means of cross validating the findings of the individual analysis.

Sweden has a public health care system and the selection of cases does not depend on socioeconomic status. The interview participation rate among the controls was low $(72 \%)$. However, the OR estimates for organic solvents and animal dust of the group analysis remained stable when subjects with missing individual exposure data were included, indicating no major selection bias for these exposures. The extensive use of next of kin interviews for cases may have hampered the validity of the individual analyses. The number of jobs reported for cases and controls in the interviews were of similar magnitude (mean number of jobs held 3.0 for cases v 3.2 for controls) and the proportion of uncertain exposure classifications did not differ systematically between cases and controls (table 2). Furthermore, a reassessment of the exposure for a sample of study subjects from the present study, as well as from studies of other haematological malignancies in southern Sweden, showed no marked difference between cases and controls for the concordance of the individual exposure classifications. ${ }^{31}$ Thus, there were no general signs of differential quality of information.

The inconsistency of the results of the individual and group analyses implies that no firm conclusion can be drawn about the association between exposure to organic solvents and $\mathrm{Ph}+\mathrm{CML}$. If causal, however, long durations of exposure to organic solvents may contribute to an important proportion of the cases of CML in southern Sweden ( $\sim 9 \%$ assuming that the true OR is 2.1 for $15-20$ years of exposure; table 3). Nevertheless, for almost all cases of $\mathrm{Ph}+\mathrm{CML}$, other explanations must be sought for.

Per-Arne Hambert assisted with exposure assessment. Siv Nilsson, Siv Hansson, and Ingegärd Nilsson performed the interviews. Gudrun Persson and Ulrika Wallin were responsible for interview administration and data entry. The project was supported by grants from the Swedish Council for Work Life Research, the Swedish Cancer Society, the Medical Faculty of Lund University, Lund University Hospital, Gunnar, Arvid and Elisabeth Nilssons Research Foundation, and PREEM Re-
search Foundation.

1 Sawyers CL. Chronic myeloid leukemia. $N$ Engl f Med 1999;340:1330-40.

2 Linet M, Cartwright R. The leukemias. In: Schottenfeld D, Fraumeni J, eds. Cancer epidemiology and prevention, 2nd ed. New York: Oxford University Press, 1996:841-92.

3 Pedersen-Bjergaard J, Brøndum-Nielsen $\mathrm{K}$, Karle $\mathrm{H}$, et al. Chemotherapy-related - and late occurring-Philadelphia chromosome in AML, ALL, and CML. Similar events related to treatment with DNA

4 Mele A, Szklo M, Visani G, et al. Hair dye use and other risk factors for leukemia and pre-leukemia: a case-control factors for leukemia and pre-leukemia:
study. Am F Epidemiol 1994;139:609-19.

5 Hayes RB, Yin SN, Dosemeci M, et al. Benzene and the dose-related incidence of hematologic neoplasms in China. 
Chinese Academy of Preventive Medicine: National Cancer Institute Benzene Study Group. 7 Natl Cancer Inst Cancer Institute

6 Raabe GK, Wong O. Leukemia mortality by cell type in petroleum workers with potential exposure to benzene. Environ Health Perspect 1996;104(suppl 6):1381-92.

7 McKinney PA, Roberts BE, O Brien C, et al. Chronic myeloid leukaemia in Yorkshire: a case control study. Acta Haematol 1990;83:35-8.

8 Kheifets LI, Afifi AA, Buffler PA, et al. Occupational electric and magnetic field exposure and leukemia. A metaanalysis. $\mathcal{F}$ Occup Environ Med 1997;39:1074-91.

9 Gentile G, Mele A, Monarco B, et al. Hepatitis B and C viruses, human T-cell lymphotropic virus types I and II, and leukemias: a case-control study. The Italian Leukemia Study Group. Cancer Epidemiol Biomarkers Prev 1996;5: 227-30.

10 Blair A, White DW. Leukemia cell types and agricultural practices in Nebraska. Arch Environ Health 1985;40:211-4.

11 Nisse C, Jouet J, Grandbastien B, et al. Chronic myeloid leukemia: a case-control study of occupational and environmenleukemia: a case-control study of occupational and environmental risk factors, 26th International Congress on Occupatid
Health, Singapore, 27th August - 1st September, 2000.

Health, Singapore, 27th August - 1st September, 2000.
12 Mauritzson N, Johansson B, Albin M, et al. A single-center population-based consecutive series of 1500 cytogenetically investigated adult hematologic malignancies: karyotypic features in relation to morphology, age and gender. Eur F Haematol 1999;62:95-102.

13 Floderus B, Persson T, Stenlund C. Magnetic-field exposures in the workplace: reference distribution and exposures in occupational groups. Int 7 Occup Environ Health 1996;2:226-38.

14 Doll R, Peto R. Mortality in relation to smoking: 20 years' observations on male British doctors. BMF 1976;2:152536.

15 Kauppinen T, Toikkanen J, Pukkala E. From crosstabulations to multipurpose exposure information systems: a new job-exposure matrix. Am f Ind Med 1998;33:409-17.

16 a new job-exposure matrix. Am find Med 1998;33:409-17. York: Wiley, 1989.

17 Checkoway H, Pearce NE, Crawford-Brown DJ. Research methods in occupational epidemiology. New York: Oxford University Press, 1989.
18 Bouyer J, Hémon D. Comparison of three methods of estimating odds ratios from a job exposure matrix in occupational case-control studies. Am f Epidemiol 1993; 137:472-81

19 Björk J, Strömberg U. Effects of systematic exposure assessment errors in partially ecologic case-control studies. Int $\mathcal{F}$ Epidemiol 2002 (in press).

20 Albin M, Björk J, Welinder $\mathrm{H}$, et al. Acute myeloid leukemia and clonal chromosome aberrations in relation to past exposure to organic solvents. Scand 7 Work Environ Health 2000;26:482-91.

21 Dosemeci M, Wacholder S, Lubin JH. Does non-differential misclassification of exposure always bias a true effect toward the null value? Am $\mathcal{F}$ Epidemiol 1990;132:746-8.

22 Ahlbom A, Day N, Feychting M, et al. A pooled analysis of magnetic fields and childhood leukaemia. $\mathrm{Br} \mathcal{F}$ Cancer 2000;83:692-8

23 Björk J, Albin M, Mauritzson N, et al. Smoking and myelodysplastic syndromes. Epidemiology 2000;11:285-91.

24 Björk J, Albin M, Mauritzson N, et al. Smoking and acute myeloid leukemia: associations with morphology and karyotypic patterns and evaluation of dose-response relations. Leuk Res 2001;25:865-72.

25 Siegel M. Smoking and leukemia: evaluation of a causal hypothesis. Am f Epidemiol 1993;138:1-9.

26 Brownson RC, Novotny TE, Perry MC. Cigarette smoking and adult leukemia. Arch Intern Med 1993;153:469-75.

27 Doll R. Cancers weakly related to smoking. $\mathrm{Br}$ Med Bull 1996;52:35-49.

28 Sandler DP. Recent studies in leukemia epidemiology. Curr Opin Oncol 1995;7:12-8.

29 Keller-Byrne JE, Khuder SA, Schaub EA. Meta-analysis of leukemia and farming. Environ Res 1995;71:1-10.

30 Bouyer J, Dardenne J, Hémon D. Performance of odds ratios obtained with a job-exposure matrix and individual exposure assessment with special reference to misclassification errors. Scand 7 Work Environ Health 1995;21:265-71.

31 Tinnerberg H, Björk J, Welinder H. Evaluation of occupational and leisure time exposure assessment in a population-based case control study on leukaemia. Int Arch Occup Environ Health 2001 (in press).

\section{Open reviewing}

Many journals, including the BMJ, have moved to a system of open reviewing, whereby authors know the names of reviewers of their papers. Research has shown that named reviews, although not of better quality than anonymous reviews, are not of worse quality either. Therefore in the interests of transparency, it seems fair to let authors know who has reviewed their paper. At Occupational and Environmental Medicine we have considered the issue carefully. There are some concerns that reviewers, especially those who are more junior, might feel intimidated and not wish to make negative comments about papers submitted by senior people in the field. On the other hand, some reviewers might hide behind the cloak of anonymity to make unfair criticisms so as to reduce the chances of publication by rivals. We have decided to introduce initially a system of open reviewing if the reviewers agree explicitly. So when a reviewer is sent a paper, he or she is asked to indicate whether we can disclose their name or not when sending the authors their comments. We will be monitoring this to see how many of our reviewers are happy to be named. If it is most of them, we will move to a system of open reviewing as the norm, with a possible "opt out" clause for reviewers. 